\title{
An Automated Tiles Defect Detection
}

\author{
V. Mohan \\ P.G. Scholar, Department Of Mechatronics \\ Jeppiaar Engineering College \\ Chennai, India
}

\author{
S. Suresh Kumar \\ P.G. Scholar, Department Of Mechatronics \\ Jeppiaar Engineering College \\ Chennai, India
}

\begin{abstract}
It presents an automatic defect identification system for detecting crack of titles from captured digital images based on defect classification and segmentation. Image classification will be used for automated visual inspection to classify defect and protects from quality one. It will be performed through textures analysis and probabilistic neural network. The textures are extracted using wavelet filters with cooccurrence features. The defect detection process involves the preprocessing, segmentation and morphological filtering to make processing system more flexible with accuracy.
\end{abstract}

\section{Keywords}

Wavelet decomposition, Cooccurrence features extraction, PNN(Probabilistic Neural Network with Radial basis Function), Segmentation and MATlab.

\section{INTRODUCTION}

The main compounds used to make the ceramic tiles are clay and other natural materials such as sand, quartz and water. Proper mixtures of all these compounds are then pressed under very high pressure into squares, rectangles, or even ovals [2] then these blocks are baked for particular time at controlled temperature.

However when it is done in large amount there may always a possibility of not uniformity hence not all the tile get the required treatment which causes the development of flaws and the type of Flaws varies depending upon which factor or factors have been compromised. As it is already mention quality control in ceramic tile manufacturing is hard, labour intensive and it is performed in a Harsh industrial environment with noise, extreme temperature and humidity [3] and inspection contains variety measurements such as colour analysis, dimension verification, and surface defect detection [3]. Hence in this paper we are proposing an efficient defect detection technique which can not only reduce the labours work but also guaranties product quality.

Throughout the last decade many kinds of defect detection systems have been developed. These systems have developed to identify the various kinds of defects on Tiles and applied to industrial process. Which provide some useful concepts for the development of the proposed algorithm. Tahir Cetin et al [4-4] presented as an examination technique based on whether there is a deformation on the material according to the sound coming from it as a result of a plastic bit hammer impact on the ceramic material. The application of the study was performed on plates made of ceramic materials. Here, it was made with the same type of model plates manufactured from the same material. The noise that would occur as a result of the impact applied on a point determined on the materials to be tested has been examined by the method of time-frequency analysis. Xien Cheng et al [5] presented a technique for ceramic bowls they utilized the bowl's curved surface unfolding model derived from helicoid unfolding method.
Jean-Luc Bouchot et al [6] analyzed the problem of finding a defect in regularly textured images with an approach based on a template matching principle.

They aim at registering patches of an input image in a defectfree reference sample according to some admissible transformations. Their proposed approach relies only on few parameters which makes it an easily adaptable algorithm for industrial applications and, above all, it avoids complex tuning of configuration parameters. Ibrahiem M.M et al [7] describe the possible use of various PNN in solving some problems arising in signal processing and pattern recognition. The main attention is devoted to application of PNN in various classification problems like: classification brain tissues in multiple sclerosis, classification image texture, classification of soil texture and EEG pattern classification. The methods for texture analysis is in transform domain like DCT, spatial domain and other available methods in detail can be found on the literature's[8][9][10][11]. The proposed paper is organized as, In section 2 provides the Proposed Methodologies, Section 3 provides experimental results and section 4 conclusion.

\section{METHODOLOGY}

\subsection{Discrete Wavelet Transform}

Wavelet Transform is a type of signal representation that can give the frequency content of the signal at a particular instant of time or spatial location. The daubechies based wavelet type transform decomposes the image into different subband images, It splits component into numerous frequency bands called subbands. They are LL, LH, HL, and HH subbands.

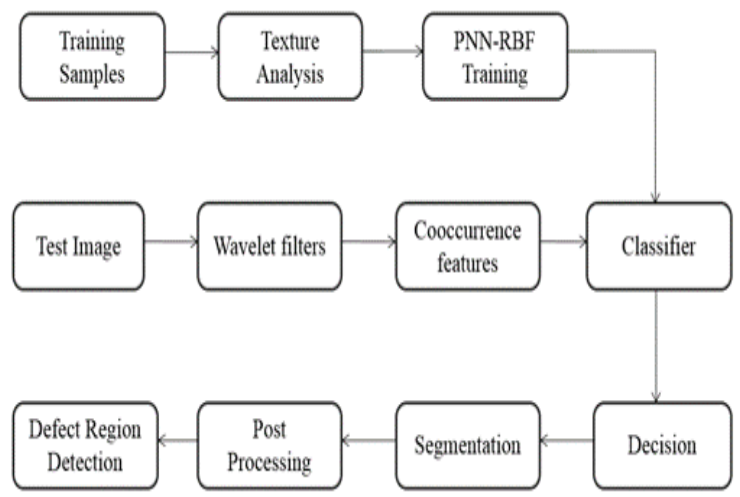

Fig 1: Proposed Block Diagram

A high-frequency subband contains the edge information of input image and LL subband contains the clear information about the image. The decomposition will be done with lifting haar and daubechies type filter to extract the texture features effectively for training process. 


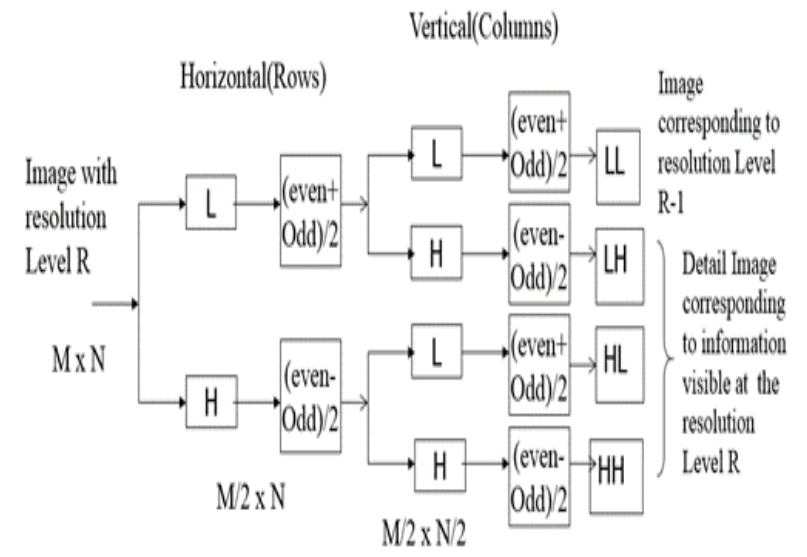

L: Low pass filter

H: High pass filter

Fig 2: Discrete Wavelet Transform Block Diagram

\subsection{Co Occurrence Matrix}

A Co-occurrence matrix (CCM) by calculating how often a pixel with the intensity (gray-level) value i occurs in a specific spatial relationship to a pixel with the value j. By default, the spatial relationship is defined as the pixel of interest and the pixel to its immediate right(horizontally adjacent), but you can specify other spatial relationships between the two pixels.

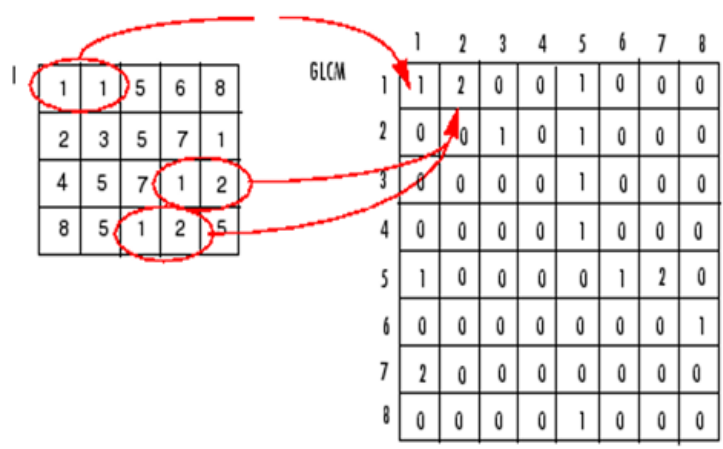

Fig 3: Matrix Generation

Each element $(i, j)$ in the resultant ccm is simply the sum of the number of times that the pixel with value i occurred in the specified spatial relationship to a pixel with value $\mathrm{j}$ in the input image. The number of gray levels in the image determines the size of the CCM.

\subsection{Haralick Features}

2.3.1 Energy: It is a measure the homogeneousness of the image and can be calculated from the normalized COM. It is a suitable measure for detection of disorder in texture image.

$$
J=\sum_{i=1} \sum_{j=1}(p(i, j))^{2}
$$

\subsubsection{Entropy: Entropy gives a measure of complexity of}

the image. Complex textures tend to have higher entropy.

$$
S=-\sum_{i=1} \sum_{j=1} p(i, j) \log (p(i, j))
$$

Where,

$$
P(i, j) \text { is the co-occurrence matrix }
$$

2.3.3 Contrast: Measures the local variations and texture of shadow depth in the gray level co-occurrence matrix.

$$
I=\sum \sum(x-y)^{2} 2 p(x, y)
$$

2.3.4 Correlation Coefficient: Measures the joint of the probability occurrence of the specified pixel pairs.

$$
\operatorname{Sum}(\operatorname{Sum}((x-2 x)(y-? y) p(x, y) / \sigma x \sigma y))
$$

\subsubsection{Homogeneity: To Measures the closeness of the}

distribution of elements in the GLCM to the GLCM of the diagonal.

$$
\operatorname{Sum}(\operatorname{Sum}(\mathrm{p}(\mathrm{x}, \mathrm{y}) /(1+[\mathrm{x}-\mathrm{y}])))
$$

\subsection{Neural Network Classifier}

Here, Supervised learning with non knowledge based classifier will be used for image classification.The neural network model PNN is used here to act as a classifier with radial basis function for network activation function. The training samples features with assigned target vectors are fed into created PNN model for supervised training to get network parameters such as node biases and weighting factors.

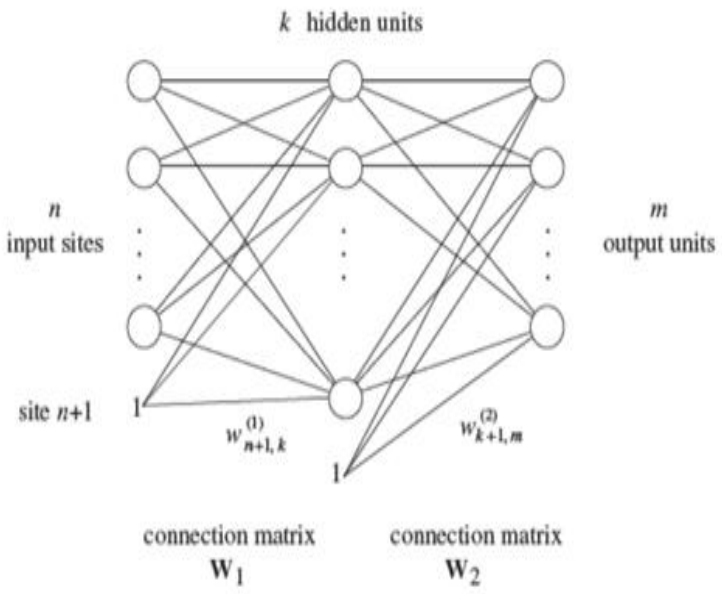

Fig 4: Neural Network Structure

Finally, test image features are simulating with trained network to make decision of steel image like normal or defective one.

\subsection{Morphological process}

Morphological operations are applied on segmented image for smoothening the brain tumor part. It processes the image based on shapes and it performs on image using structuring element.Dilation and erosion process will be used to enhance (smoothening)the tumor region by removing the unwanted pixels from outside region of tumor part. 


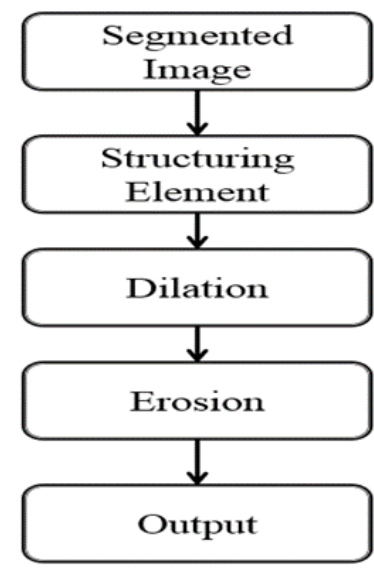

Fig 5: Flow Chart of Morphological Process

Dilation: It is the process of adding a pixel at object boundary based on structuring element.

$$
\text { Dilation (D) = I (bit Xor) Se }
$$

Erosion : It is to remove the pixel from the object boundary depends on structuring element.

$$
\text { Erosion }(\mathrm{E})=\mathrm{I}(\text { bit Xnor) Se }
$$

I - Input Image, $\mathrm{Se}=$ Line Structuring Element

\section{EXPERIMENTAL RESULTS}

The analysis of the proposed algorithm is performed for different number of training and testing data sized and also the comparison with the existing methods [2] are presented in this section.

Table 1. Detection rate comparison

\begin{tabular}{|c|c|c|}
\hline Number of Tiles & Previous Work & Proposed Work \\
\hline 10 & $100 \%$ & $100 \%$ \\
\hline 20 & $90 \%$ & $100 \%$ \\
\hline 30 & $90 \%$ & $97.656 \%$ \\
\hline 40 & $92.5 \%$ & $96.875 \%$ \\
\hline 50 & $92 \%$ & $96.354 \%$ \\
\hline Average & $93 \%$ & $98.177 \%$ \\
\hline
\end{tabular}

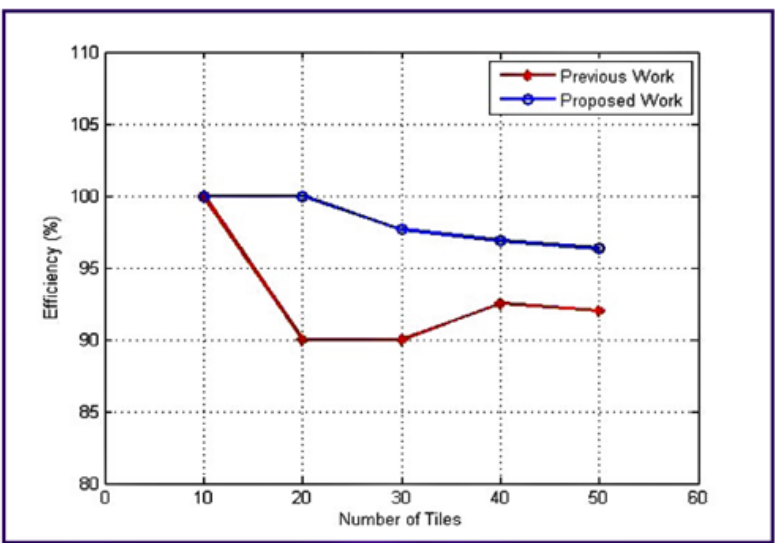

Fig 6: Comparison graph for table 1. The graph shows that the proposed system increases the overall detection rate by $\mathbf{5 \%}$
Table 2. Detection time (in seconds) comparison for different number of tiles

\begin{tabular}{|c|c|c|}
\hline $\begin{array}{c}\text { Number of } \\
\text { Tiles }\end{array}$ & Previous Work & Proposed Work \\
\hline 10 & 3.538866 & 1.5336 \\
\hline 20 & 7.55837 & 2.9706 \\
\hline 30 & 11.145318 & 5.8589 \\
\hline 40 & 15.150612 & 7.3691 \\
\hline 50 & 19.242661 & 8.7952 \\
\hline
\end{tabular}

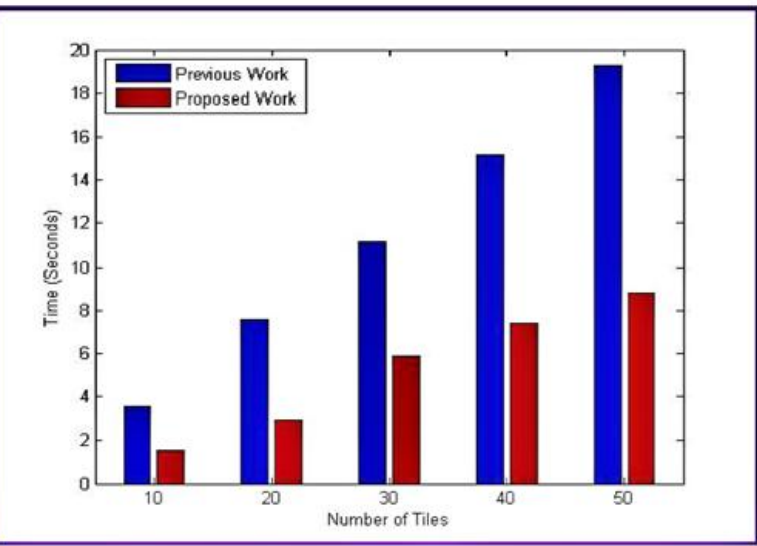

Fig 7: Comparison graph for table 2. The graph shows that the proposed system takes half of the time taken by previous algorithm

Table 3. Detection rate comparison for individual defects

\begin{tabular}{|c|c|c|}
\hline Defect & Previous Work & Proposed Work \\
\hline Crack & $86.49 \%$ & $100 \%$ \\
\hline Blob & $87.50 \%$ & $100 \%$ \\
\hline Edge & $96.77 \%$ & $97.92 \%$ \\
\hline Corner & $93.55 \%$ & $95.83 \%$ \\
\hline
\end{tabular}

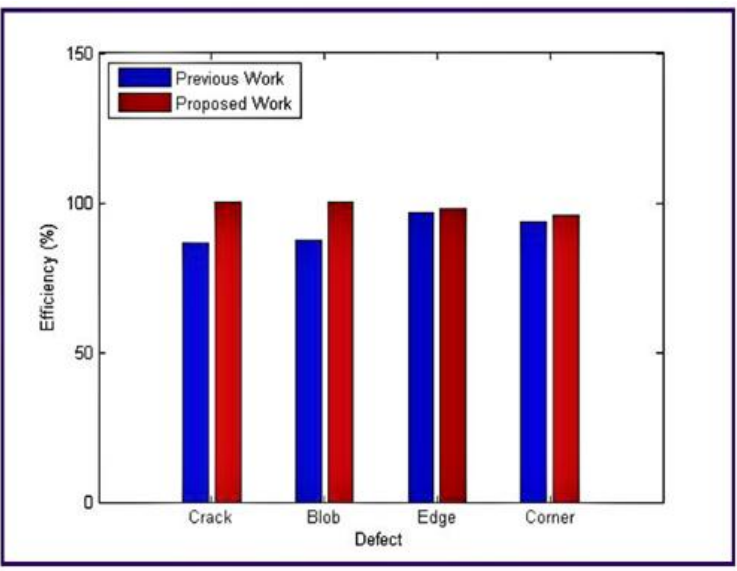

Fig 8: Comparison graph for table 3. The graph shows that the proposed algorithm out performs the previous algorithm in every type of defect detection 


\section{CONCLUSION}

In this paper, tiles defect identification technique is proposed and simulated with in depth analysis and comparison with recently proposed technique. The analysis shows that proposed algorithm increases detection rate over 5\% than the previous algorithm and it also slash down the detection time to half of the previous algorithm which is a necessary requirement for higher production rate.

The another benefit of the proposed algorithm that it does not required exact mathematical modeling of the defects so it can detect a Varity of known \& unknown characteristics of tiles simultaneously. However in future some other classifier improvement may also be applied like pre-clustering, feature optimization and weight estimation which can further improve the efficiency of the system.

\section{ACKNOWLEDGMENT}

The authors would like to thank Dr.S.Rajesh, Associate Professor, Department of Mechanical Engineering, Jeppiaar Engineering College, Chennai, for his valuable guidance and continuous support in making this paper.

\section{REFERENCES}

[1] R.C. Gonzalez, R. E. Woods, "Digital Image Processing", Pearson Education (Singapore), Pte. Ltd., Indian Branch, 482 F.I.E, Partapgang, 20052006.

[2] Md. Maidul Islam, Md. RowshanSahriar and Md. Belal Hossain -An Enhanced Automatic Surface and Structural Flaw Inspection and Categorization using Image Processing Both for Flat and Textured Ceramic Tiles\|, International Journal of Computer Applications (0975 - 888) Volume 48- No.3, June 2012.

[3] H. Elbehiery, A. Hefnawy, and M. Elewa - Surface Defects Detection for Ceramic Tiles Using Image Processing and Morphological Techniquesll, PROCEEDINGS OF WORLD ACADEMY OF SCIENCE, ENGINEERING AND TECHNOLOGY VOLUME 5 APRIL 2005 ISSN 1307-6884.

[4] Tahir Cetin - The Defect Detection in Ceramic Materials Basedon Time-Frequency Analysis by Using the Methodof Impulse Noisell, ARCHIVES OF ACOUSTICS DOI: 10.2478/v10168-011-0007-y36, 1, 77-85 (2011).

[5] Xien Cheng and JinghuaZheng - Novel Approach of Surface Unfolding for Ceramic Bowlsl, I.J. Engineering and Manufacturing 2011, 3, 1-6.

[6] Jean-Luc Bouchot, GernotStubland Bernhard Moser - A template matching approach based on the discrepancynorm for defect detection on regularly textured surfaces\|, https://www.academia.edu.

[7] Ibrahiem M.M. El Emary and S. Ramakrishnan -On the Application of Various Probabilistic Neural Networks in Solving Different Pattern Classification Problemsll, World Applied Sciences Journal 4 (6): 772-780, 2008 ISSN 1818-4952.

[8] Yu Tao, VallipuramMuthukkumarasamy, BrijeshVerma and Michael Blumenstein -A Texture Feature Extraction Technique Using 2D- DFT and Hamming Distancell, Computational Intelligence and Multimedia Applications, 2003. ICCIMA 2003. Proceedings. Fifth International Conference on 27-30 Sept. 2003.

[9] Yang Mingqiang, KpalmaKidiyo and Ronsin Joseph -A Survey of Shape Feature Extraction Techniquesll, IETRINSA, UMR-CNRS 6164, 35043 Rennes, Shandong University, 250100, Jinan.

[10] Roberto Márcio de Andrade and Alexandre Carlos Eduardo - Methodology for Automatic Process of the Fired Ceramic Tile's Internal Defect Using IR Images and Artificial Neural Networkl, Methodology for Automatic Process of the Fired Ceramic Tile's Internal Defect Using IR Images and Artificial Neural Network January-March 2011, Vol. XXXIII, No. 1 / 67.

[11] AndrzejMaterka and Michal Strzelecki -Texture Analysis Methods - A Reviewll, A. Materka, M. Strzelecki, Texture Analysis Methods - A Review, TechnicalUniversity of Lodz, Institute of Electronics, COST B11 report, Brussels 1998. 